wirkung durch Anwendung gerichteten Lichts gesteigert wird, das mit Hilfe der als Zylinderlinsen wirkenden Proberöhrchen auf das lichtempfindliche Material konzentriert wird, während die im trüben Medium gestreuten Strahlen durch eine vor dem lichtempfindlichen Material angeordneten Spaltblende fast vollständig abgefangen werden.

\section{h) Kolloidmühlen.}

D.R.P. 504196 vom 22. März 1925. Röhm \& Haas A. G., Darmstadt.

Kolloidschlägermühle zum Mahlen pastöser oder breiiger Stoffe, dad. gek., daß an das Gehäuse eine oder mehrere mit Hähnen versehene Leitungen für die Zuführung von Luft oder Gasen angeschlossen sind.

D.R.P. 504598 vom 22. Mai 1928. Dr. August Chwala, Wien.

Verfahren zur mechanischen Dispergierung von in Wasser schwerlöslichen oder unlöslichenVerbindungen, ausgenommen von Erdalkali- und Schwermetallsalzen der Phosphor- und Arsensäuren, dad. gek., daß als Peptisatoren Alkalisalze der Arsensäuren oder Phosphorsäuren verwendet werden, die aus den Orthosäuren durch Wasserabspaltung entstehen oder so entstanden gedacht werden können, d. h. im Verhältnis zu diesen Orthosäuren wasserärmer sind.

\section{i) Verschiedenes.}

D.R.P. 502550 vom 31. Januar 1926. Firma Dr. A. Schmitz, Düsseldorf-oberkassel.

Mittel zum Reibechtmachen reibunechter Austärbungen auf pflanzlichen oder tierischen Fasern, bestehend aus einer in heißer wässeriger Lösung anzuwendenden, möglichst innigen Mischung von etwa drei Gewichtsteilen eines nativen Eiweißstoffes, insbesondere Kasein, mit etwa zwei Gewichtsteilen eines borsauren Alkali- oder Ammonsalzes und etwa fünf Gewichtsteilen eines Ammoniumsalzes einer anorganischen oder organischen Säure.

D.R.P. 502553 vom 8. Dezember 1928, Hans Burmeister, Berlin.

Vorrichtung zum Mischen von Gasen und Flüssigkeiten, mittels eines körnigen oder feinporigen Verteilungskörpers, dad. gek., daß der Durchgangswiderstand längs der Behälterwandung größer gehalten ist, als der Durchgangswiderstand durch den Verteilungskörper selbst, z. B. durch Bildung von tiefen Rillen in der inneren Behälterwandung oder durch Einbau vorspringender Ringe.

D.R.P. 502913 vom 31. Juli 1926. Karl Le Hamec, Krefeld.

Verfahren zur Trocknung von für landwirtschaftliche oder ähnliche Zwecke verwertbaren Schlamm, dad. gek., daB derSchlamm unter beständigerBewegung und in dünner Schicht ausgebreitet und unter kontinuierlichem Durchgang durch eine Rieseltrocknungsvorrichtung mit erwärmten Gasen oder überhitztem Dampf behandelt wird, derart, daß man das Trocknungsmittel an verschiedenen Stellen des Trocknungsraumes durch die dünne Schlammschicht hindurchtreten läßt und für sofortige Entfernung des mit Feuchtigkeit beladenen Trocknungsmittels Sorge trägt, wobei eine Erhitzung des Schlammes auf Tempera- turen, welche die humusbildenden Bakterien schädigen, zu vermeiden ist.

D.R.P. 503047 vom 18. Oktober 1927. Paul Rohland, Dreiwerden, Sa.

Verfahren zur Herstellung von Satinglanzweiß, Kalk-Tonerdelacken und anderen Kalziumaluminaten enthaltenden Streichfarben in einer Mühle, dad. gek., daß ihre Bildung aus der Reaktionsmischung in schnellaufenden Mühlen, z. B. Mühlen nach P. 488354 und 502484, oder sogenannten Kolloidmühlen in ununterbrochenem Durchfluß erfolgt.

D.R.P. 505267 vom 20. Oktober 1927. The Silica Gel Corporation, Baltimore (V.S.A.).

Verfahren zur Kälteerzeugung, bei dem ein Kältemittel von einer feinporigen, festen Substanz im dampfförmigen Zustand adsorbiert und aus ihr wieder ausgetrieben wird, dad. gek., daß die Adsorptionswärme in einem oder mehreren Adsorbern für die Reaktivierung in anderen Adsorbern nutzbar gemacht wird.

D.R.P. 503048 vom 13. April 1929. Jos. Scholz G. m. b. H., Mainz.

Verfahren zur Herstellung von Siegellack in mehr oder weniger fein verteilter Form ohne Verwendung von Gießformen, dad. gek., daß man den Siegellack aus einer Tropfvorrichtung in eine Kühlflüssigkeit, in welcher der Siegellack unlöslich ist, vorteilhaft Wasser, abtropfen läßt.

D.R.P. 503351 vom 25. März 1926. I. G. Farbenindustrie A. G., Frankfurt a. M.

Verfahren zur Herstellung haltbarer Kohlesuspensionen durch mechanisches Dispergieren von Kohle in Alkoholen, gek. durch den Zusatz von alkohollöslichen anorganischen oder organischen Basen.

D.R.P. 503199 vom 28. September 1928 (Zusatz zum P. 358700 ). I. G. Farbeninstrie, A. G. Frankfurt a. M.

Verfahren zur Herstellung von kolloidalem Schwefel gemäß P. 358700, dad. gek., daß man die Zersetzung der Polysulfidlösung unter Druck zweckmäßig in einer Abtreibekolonne, mit gespanntem Wasserdampf vornimmt.

D.R.P. 503496 vom 23. Juni 1927. Kali-Chemie A. G. Berlin.

Verfahren zur Gewinnung von Bariumhydroxyd durch Hydrolyse von schwer hydrolysierbaren Bariumsilikaten, dad. gek., daß man die Bariumsilikate in einer Kornfeinheit anwendet, bei welcher die Korngröße im wesentlichen unterhalb $1 / 10 \mathrm{~mm}$ bis zur praktisch kolloidalen Feinheit liegt.

D.R.P. 503423 vom 30. Oktober 1928; (Zusatz zum P. 472346). Chem. Fabrik vorm. Sandoz, Basel (Schweiz).

Verfahren zur Herstellung von haltbaren übersättigten Kalziumglukonatlösungen nach P. 472346, dad. gek., daß man konzentrierte Kalziumglukonatlösungen, denen andere therapeutisch verwendbare lösliche Stoffe zugesetzt sind, heiß abfüllt und unter völligem AbschluB, gegebenenfalls in Abständen wiederholt, so lange erhitzt, bis keine Kristallbildung mehr eintritt.

\section{Berichtigung}

Als Verfasser der Arbeit: Studien über die Bildung von Liesegang'schen Ringen Koll.-Zeitschr. 54, 190 (1931) soll es heißen Mrs. N. R. D har statt Miss S. Ro y. 